\title{
MODULAR CATEGORIES, INTEGRALITY AND EGYPTIAN FRACTIONS
}

\author{
PAUL BRUILLARD AND ERIC C. ROWELL
}

(Communicated by Harm Derksen)

\begin{abstract}
It is a well-known result of Etingof, Nikshych and Ostrik that there are finitely many inequivalent integral modular categories of any fixed rank $n$. This follows from a double-exponential bound on the maximal denominator in an Egyptian fraction representation of 1. A naïve computer search approach to the classification of rank $n$ integral modular categories using this bound quickly overwhelms the computer's memory (for $n \geq 7$ ). We use a modified strategy: find general conditions on modular categories that imply integrality and study the classification problem in these limited settings. The first such condition is that the order of the twist matrix is $2,3,4$ or 6 , and we obtain a fairly complete description of these classes of modular categories. The second condition is that the unit object is the only simple non-self-dual object, which is equivalent to odd-dimensionality. In this case we obtain a (linear) improvement on the bounds and employ number-theoretic techniques to obtain a classification for rank at most 11 for odd-dimensional modular categories.
\end{abstract}

\section{INTRODUCTION}

The problem of classifying low-rank modular categories has its roots in the classification problem for rational conformal field theories going back to the 1980s (predating the definition of modular category [25]). Currently, the most complete results are in [23, where unitary modular categories of rank at most 4 are classified. More generally, Ostrik classified ribbon fusion categories of rank at most 3 [21] and fusion categories of rank at most 2 [20].

By a generalized form of Ocneanu rigidity [7, Prop. 2.31] one may classify modular categories of a given rank up to finite ambiguity by classifying their Grothendieck semirings. In [13] such an approach yielded a classification (up to Grothendiecksemirings) of modular categories of rank at most 5 with the property that some object is not isomorphic to its dual object.

Wang has conjectured that there are only finitely many inequivalent modular categories of each rank (see [23, Conjecture 6.1]), and the results mentioned above bear this out for rank at most 4 and for rank at most 5 in case some object is non-self-dual. The most general class of modular categories for which Wang's conjecture has been verified is for weakly integral categories, that is, categories $\mathcal{C}$ with FPdim $(\mathcal{C}) \in \mathbb{N}$ [7, Prop. 8.38]. The proof relies upon a classical result of Landau

Received by the editors December 3, 2010 and, in revised form, December 8, 2010. 2010 Mathematics Subject Classification. Primary 18D10; Secondary 16T05, 11Y50. 
[14] that the Diophantine equation

$$
1=\sum_{i=1}^{n} \frac{1}{x_{i}}
$$

has finitely many solutions with $x_{i} \in \mathbb{N}$. Such solutions are Egyptian fraction representations of 1 which are of independent interest in combinatorial number theory (see [12, Section D11] and [19, Seq. A002966]). Moreover the number of solutions to (1.1) is at least exponential in $n$ since $\frac{1}{x}=\frac{1}{x+1}+\frac{1}{x^{2}+x}$ so that each (non-constant) $n$-term Egyptian fraction representation of 1 leads to at least $2(n+1)$-term representations. In [13] a computational approach to classifying integral modular categories (that is, with $\operatorname{FPdim}(X) \in \mathbb{N}$ for all objects $X$ ) of rank $n$ is suggested using two facts:

(1) [6, Lemma 1.2]: $\sqrt{\frac{\text { FPdim(e) }}{\operatorname{FPdim}\left(X_{i}\right)}} \in \mathbb{N}$ for all simple $X_{i}$;

(2) [3]: $\operatorname{dim}(\mathcal{C}) \leq u_{n}$, where $u_{n}$ is inductively defined by $u_{1}:=1$ and $u_{k}:=$ $u_{k-1}\left(u_{k-1}+1\right)$.

As $u_{n}$ is doubly exponential in $n$, a direct search for solutions by computer quickly becomes infeasible.

We attempt to circumvent this computational obstacle by finding interesting conditions on modular categories that imply integrality and then using these conditions to simplify the classification problem for these classes of categories. Specifically, we have:

Theorem. Suppose $\mathcal{C}$ is a modular category such that either

(a) the twist matrix $T$ satisfies $T^{N}=I$ for $N \in\{2,3,4,6\}$ or

(b) the only simple object in $\mathcal{C}$ satisfying $X \cong X^{*}$ is the unit object $\mathbf{1}$.

Then $\mathrm{C}$ is integral.

Statement (a) is proved below in Theorem 3.1 and was inspired by Davydov, who posed the question for $N=2$ to the second author. Statement (b) is 13 , Theorem 2.2] and follows from a Galois theory argument.

We are interested in classifying categories with one of these two properties (a) or (b). We obtain a fairly explicit description of modular categories with property (a) in Theorem 3.2, Modular categories with property (b) of rank at most 11 are shown to be pointed in Theorem 4.5, that is, $\operatorname{FPdim}\left(X_{i}\right)=1$ for each simple object $X_{i}$. While property (b) may seem to be a rare condition at first glance, one can show (see Prop. 4.3) that it is equivalent to the condition that FPdim(e) is odd.

The remainder of the paper is structured as follows. In Section 2 we collect together some notation and useful facts about modular categories. We address the classification problem for modular categories having property (a) or (b) in Sections 3 and 4 respectively, and give some perspectives and futher directions in Section 5.

\section{Notation}

A modular category $\mathcal{C}$ is a non-degenerate braided, balanced fusion category (see [1] or 22 for the complete axiomatic definition). In this section we establish notation and describe some of the algebraic data and relations coming from the axioms of modular categories.

We shall typically adopt the notation and normalizations of [18] for the data of a modular category. The fusion coefficients are $N_{i, j}^{k}:=\operatorname{dim} \operatorname{Hom}\left(X_{i} \otimes X_{j}, X_{k}\right)$, where 
$\mathbf{1}=X_{0}, \ldots, X_{n-1}$ are the (isomorphism classes of) simple objects, the number of which $(n)$ is called the rank of $\mathcal{C}$. The diagonal twist matrix $T_{i j}:=\delta_{i j} \theta_{i}$ has finite order and the $S$-matrix is normalized so that $S_{00}=1$. We will denote by $d_{i}$ the dimension of the simple object $X_{i}$, i.e. $d_{i}=S_{i 0}=\operatorname{dim}\left(X_{i}\right)$. Defining the Gauss sums by $p_{+}=\sum_{i} \theta_{i}\left(d_{i}\right)^{2}$ and $p_{-}=\overline{p_{+}}$we have $(S T)^{3}=p_{+} S^{2}, S^{2}=$ $p_{+} p_{-} C$, where $C_{i j}=\delta_{i j^{*}}$ is the (involutive) charge conjugation matrix, which commutes with $T$. In particular, $(S, T)$ give rise to a (projective) representation of the modular group $S L(2, \mathbb{Z})$. We define the fusion matrices $\left(N_{i}\right)_{j k}:=N_{i k}^{j}$ and denote by $\mathrm{FPdim}\left(X_{i}\right)$ the largest eigenvalue of $N_{i}$, i.e. the FP-dimension of $X_{i}$. The global dimension of $\mathcal{C}$ is $\operatorname{dim}(\mathcal{C})=\sum_{i}\left(d_{i}\right)^{2}$, and the global $F P$-dimension is $\mathrm{FPdim}(\mathcal{C}):=\sum_{i} F P \operatorname{dim}\left(X_{i}\right)^{2}$. If $\mathrm{FPdim}(\mathcal{C})=\operatorname{dim}(\mathcal{C})$, then $\mathcal{C}$ is said to be pseudounitary. The Verlinde formula relates the fusion coefficients to the $S$-matrix entries:

$$
N_{i j}^{k}=\sum_{r} \frac{S_{i r} S_{j r} \overline{S_{k r}}}{\operatorname{dim}(\mathcal{C}) S_{0 r}} .
$$

A pair of matrices $(S, T)$ satisfying the above relations such that the right-hand side of (2.1) is a non-negative integer for all $i, j, k$ is called a modular datum ([9]). A modular category $\mathcal{C}$ with corresponding $S$ and $T$ matrices is called a categorification of $(S, T)$.

A category $\mathcal{C}$ is called integral if $\operatorname{FPdim}\left(X_{i}\right) \in \mathbb{N}$ for all simple objects $X_{i}$. All categories encountered in this work will be integral and hence pseudo-unitary so that we may assume that $\mathrm{FPdim}\left(X_{i}\right)=d_{i}$ and $\mathrm{FPdim}(\mathrm{C})=\operatorname{dim}(\mathrm{C})$ by [7, Prop. 8.23, 8.24].

The entries of $N_{i}, S$ and $T$ satisfy further relations:

$$
p_{+} p_{-}=\operatorname{dim}(\mathcal{C})
$$

and

$$
\theta_{i} \theta_{j} S_{i j}=\sum_{k} N_{i^{*} j}^{k} \theta_{k} d_{k}
$$

Since dim is a character of the Grothendieck semiring of $\mathcal{C}$, we have

$$
\sum_{k} N_{i j}^{k} d_{k}=d_{i} d_{j}
$$

The (second) FS-indicator is defined to be

$$
\nu_{k}=\frac{1}{\operatorname{dim}(\mathcal{C})} \sum_{i, j} N_{i, j}^{k} d_{i} d_{j}\left(\frac{\theta_{i}}{\theta_{j}}\right)^{2}
$$

and satisfies

$$
\nu_{k}= \begin{cases}0 & X_{k} \approx X_{k}^{*}, \\ \pm 1 & X_{k} \cong X_{k}^{*}\end{cases}
$$

More generally the $n$-th FS-indicator $\nu_{k}^{(n)}$ may be defined for simple objects in modular categories in an analogous way ([17, Theorem 7.5]). The quantity FSexp $(\mathcal{C})$ is the smallest integer $m>0$ such that $\nu_{k}^{(m)}=d_{k}$ for all simple $k$. For modular categories, $F \operatorname{Sexp}(\mathcal{C})$ coincides with the order of the $T$ matrix $([17$, Theorem 7.7]). 


\section{LOW-ORDER TWIST MATRICES}

In this section we study modular categories with twist matrix of order $2,3,4$ or 6 of arbitrary rank. Our first result is:

Theorem 3.1. Suppose that $T$ is the twist matrix of a modular category $\mathcal{C}$ such that $T^{N}=I$ for $N \in\{2,3,4,6\}$. Then $\mathcal{C}$ is integral.

Proof. First we observe that by [18, Prop. 5.7] the entries of the $S$-matrix for $\mathcal{C}$ must lie in $\mathbb{Q}\left(\theta_{1}, \ldots, \theta_{n-1}\right)=\mathbb{Q}\left(\zeta_{N}\right)$, where $\zeta_{N}$ is a primitive $N$ th root of unity. Since the $s_{i j}$ are algebraic integers and $\varphi(N) \leq 2$ (Euler's totient $\varphi$ ), if $S_{i j} \in \mathbb{R}$, then $S_{i j} \in \mathbb{Z}$. In particular some column of the $S$-matrix must be an integer multiple of the vector of $F P$-dimensions (since $\mathcal{C}$ is modular), and so $\operatorname{FPdim}\left(X_{i}\right)$ are rational integers.

We now characterize modular categories with $T^{N}=I$ for $N \in\{2,3,4,6\}$ in the following:

Theorem 3.2. Suppose $\mathcal{C}$ is a modular category such that $T^{N}=I$. Then:

(a) If $N=2$, then $\mathcal{C}$ is braided tensor equivalent to a subcategory of $\operatorname{Rep}\left(D^{\omega} G\right)$, where $G$ is an abelian 2-group of exponent 2 and $\operatorname{dim}(\mathrm{C})=2^{2 s}$ and moreover, $\mathcal{C}$ is pointed.

(b) If $N=3$, then $\mathcal{C}$ is braided tensor equivalent to a subcategory of $\operatorname{Rep}\left(D^{\omega} G\right)$, where $G$ is a 3-group of exponent 3 .

(c) If $N=4$, then $\mathcal{C}$ is braided tensor equivalent to a subcategory of $\operatorname{Rep}\left(D^{\omega} G\right)$, where $G$ is a 2-group of exponent 2 or 4 .

(d) If $N=6$, then $\mathrm{C}$ is solvable and hence weakly group-theoretical.

Proof. If $T^{2}=I$, then each $X_{k}$ is self-dual since the entries of the $S$-matrix are real. Computing the FS-indicator we have

$$
\nu_{k}=\frac{1}{\operatorname{dim} \mathcal{C}} \sum_{i, j} N_{i j}^{k} d_{i} d_{j}\left(\frac{\theta_{i}}{\theta_{j}}\right)^{2}= \pm 1
$$

for all $k$. But $\left(\frac{\theta_{i}}{\theta_{j}}\right)^{2}=1$ by assumption and $\sum_{i} N_{i j}^{k} d_{i}=d_{k} d_{j}$ (as $N_{i j}^{k}$ is totally symmetric in the self-dual case), so we may simplify:

$$
\nu_{k}=\frac{1}{\operatorname{dim} \mathrm{C}} \sum_{j} d_{k} d_{j}^{2}=d_{k} .
$$

But $\nu_{k}= \pm 1$ and $d_{k}>0$, so this implies $d_{k}=1$ for all simple objects $X_{k}$. We must then have $X_{k}^{\otimes 2} \cong \mathbf{1}$ as well. Thus $\mathcal{C}$ is pointed and has the same fusion rules as the group $\mathbb{Z}_{2}^{m}$, where $2^{m}$ is both the rank and global dimension of $\mathcal{C}$. Now since $p_{+} \in \mathbb{R}$ we have $p_{+}=p_{-} \in \mathbb{Z}$ so that by (2.2) we must have $\operatorname{dim}(\mathcal{C})=2^{m}=\left(p_{+}\right)^{2}$ and hence $m=2 s$. Since $\mathcal{C}$ is braided tensor equivalent to a subcategory of $Z(\mathcal{C}) \cong \operatorname{Rep}\left(D^{\omega} G\right)$ (by [4, Theorem 6.10]) we must have that $G$ is an elementary abelian 2-group.

For (b) and (c) with $N=3$ (resp. 4) we use [17, Theorem 8.4] to conclude that $\operatorname{dim}(\mathcal{C})=p^{t}$, where $p=3$ (resp. 2). In particular $\mathcal{C}$ is braided and nilpotent and hence group-theoretical by [4, Theorem 6.10]. Thus we conclude that $\mathcal{C}$ is braided tensor equivalent to a subcategory of $Z(\mathcal{C}) \cong \operatorname{Rep}\left(D^{\omega} G\right)$, where $G$ is a $p$-group, and since $\mathrm{FSexp}(\mathcal{C})=\mathrm{FSexp}(Z(\mathcal{C}))=3$ (resp. 4) by [17, Corollary 7.8], $G$ must have exponent 3 (resp. 2 or 4 as the exponent of $G$ must divide that of $\operatorname{Rep}\left(D^{\omega} G\right)$ ). 
For (d) with $N=6$, [17, Theorem 8.4] implies that $\operatorname{dim}(\mathcal{C})=2^{a} 3^{b}$ with $a, b>0$, and so by [8, Theorem 1.6] $\mathcal{C}$ is solvable and, in particular, weakly group-theoretic.

Remark 3.3.

- In principle, Theorem 3.2 (a),(b),(c) can be used to completely classify modular categories with twist matrix of orders 2,3 and 4 .

- Theorem 3.2(d) is sharp in the sense that we cannot conclude that $\mathcal{C}$ is group-theoretical, as [10, Example 5.3(a)] shows that non-group-theoretical modular categories with $T^{6}=I$ exist.

- In [2, Prop. 5.1] an infinite family of modular data $(S, T)$ depending on an integer $k \geq 0$ is described with $S_{i j} \in \mathbb{Z}$ for all $i, j$ and $T^{2}=I$. Theorem 3.2 (a) shows that this family is not categorifiable for $k \geq 1$.

\section{INTEGRALITY AND EGYPTIAN FRACTIONS}

In this section we classify integral modular categories of rank at most 6 and maximally non-self-dual modular categories of rank at most 11.

If $\mathcal{C}$ is a (pseudo-unitary) integral modular category with dimensions of simple objects $d_{1}, d_{2}, \ldots, d_{n}=\operatorname{dim}(\mathbf{1})=1$, then by [6. Lemma 1.2] the numbers $x_{i}=$ $\operatorname{dim}(\mathcal{C}) /\left(d_{i}\right)^{2}$ are integers and so satisfy (1.1). In [13, Lemma 2.3] it is shown that after relabelling if necessary so that $x_{1} \leq x_{2} \leq \cdots \leq x_{n}=\operatorname{dim}(\mathrm{C})$, then:

$$
k \leq x_{k} \leq u_{k}(n-i+1) \text { for all } 1 \leq k \leq n,
$$

where $u_{1}=1, u_{k}=u_{k-1}\left(u_{k-1}+1\right)$. These bounds rely upon two classical results in $\left[14\right.$ and [3]. Notice also that $x_{n} / x_{i}=\left(d_{i}\right)^{2}$ is a perfect square for each $i$. The trivial solution $x_{1}=\cdots=x_{n}=n$ corresponds to a pointed modular category of rank $n$ as then $d_{i}=1$ for all $i$. Our basic algorithm for finding $x_{1} \leq \cdots \leq x_{n}$ satisfying these conditions is as follows:

\section{Algorithm 4.1.}

(1) Form the set of pairs $S_{2}:=\left\{\left(j, j \cdot i^{2}\right): 2 \leq j \leq n, 1 \leq i \leq \sqrt{u_{n} / j}\right\}$. These are the possible pairs $\left(x_{1}, x_{n}\right)$.

(2) Having determined $S_{k}$ for some $2 \leq k \leq n-2$, let $S_{k+1}$ be the set of $(k+1)$-tuples $\left(j_{1}, \ldots, j_{k-1}, J, j_{k}\right)$ such that
(a) $\left(j_{1}, \ldots, j_{k}\right) \in S_{k}$,
(b) $\max \left(j_{k-1}, k\right) \leq J \leq \min \left(j_{k}, u_{k}(n-k+1)\right)$,
(c) $\sqrt{j_{k} / J} \in \mathbb{Z}$ and
(d) $\frac{1}{J}+\sum_{i=1}^{k} \frac{1}{x_{i}} \leq 1$.

(3) The solution set $S_{n}$ is the set of $n$-tuples $\left(j_{1}, \ldots, j_{n-2}, J, j_{n-1}\right)$, where
(a) $\left(j_{1}, \ldots, j_{n-1}\right) \in S_{n-1}$,
(b) $\max \left(j_{n-2}, n-1\right) \leq J \leq \min \left(j_{n-1}, 2 u_{n-1}\right)$,
(c) $\sqrt{j_{n-1} / J} \in \mathbb{Z}$ and
(d) $\frac{1}{J}+\sum_{i=1}^{n-1} \frac{1}{x_{i}}=1$.

For $n=5$ and $n=6$ the solutions are $\{(5,5,5,5,5),(2,8,8,8,8)\}$ and

$$
\{(6,6,6,6,6,6),(3,3,12,12,12,12),(4,4,4,9,9,36)\}
$$

respectively. These computations were carried out in a few hours on a desktop computer using Maple, and we have: 
Theorem 4.2. Any integral modular category of rank at most 6 is pointed.

Proof. For rank at most 5 this result is known; see [23] and [13. For rank 6 we need only show that no modular category may have simple dimensions $(1,1,1,1,2,2)$ or $(1,2,2,3,3,3)$ corresponding to the non-pointed solutions above. The first case is eliminated by [15, Prop. 4.11]: integral modular categories $\mathcal{C}$ with $\mathrm{FPdim}(\mathcal{C})=2^{2} 3$ must be pointed. On the other hand, a fusion category of dimension 36 must be solvable and contain a non-trivial invertible object by [8, Prop. 8.2], which eliminates the second solution.

We remark that there is a non-pointed integral modular category of rank 8 , namely $\operatorname{Rep}\left(D\left(S_{3}\right)\right)$, the representation category of the double of the symmetric group $S_{3}$. We expect that this is the smallest possible rank for non-pointed integral modular categories. Unfortunately for $n=7$ one has $u_{7} \approx 10^{13}$, and attempts to implement Algorithm 4.1 quickly overwhelm a computer's memory. This motivates passing to a restricted class of modular categories that enjoy integrality.

With a view towards improving the upper bound $u_{n}$ above, suppose that $\mathcal{C}$ is a maximally non-self dual (MNSD) modular category; i.e. each non-trivial simple object $X_{i}$ satisfies $X_{i} \neq X_{i}^{*}$. Then $\mathcal{C}$ is integral with rank $n=2 k+1$ by [13, Theorem 2.2]. Since $d_{i}=\operatorname{dim}\left(X_{i}\right)=\operatorname{dim}\left(X_{i}^{*}\right)$ we may label the dimensions of simple objects: $d_{1}, d_{1} \ldots, d_{k}, d_{k}, d_{k+1}=\operatorname{dim}(\mathbf{1})=1$ so that $\operatorname{FPdim}(\mathcal{C})=1+$ $2 \sum_{i=1}^{k} d_{i}^{2}$ is odd. After reordering the $k+1$ integers $x_{i}:=\operatorname{dim}(\mathcal{C}) / d_{i}^{2}$ so that $x_{1} \leq x_{2} \leq \cdots \leq x_{k} \leq x_{k+1}=\operatorname{dim}(\mathrm{C})$ they satisfy the special case of (1.1):

$$
\frac{1}{x_{k+1}}+\sum_{i=1}^{k} \frac{2}{x_{k}}=1 .
$$

The maximal non-self-dual condition may seem somewhat exceptional, but it follows from [13, Theorem 2.2], [17, Corollary 8.2(ii)] and [5, Corollary 2.33] that:

Proposition 4.3. Let $\mathcal{C}$ be a modular category of rank $n$. Then the following are equivalent:

(1) $\mathcal{C}$ is maximally non-self-dual.

(2) $\mathrm{FPdim}(\mathrm{C})$ is odd.

(3) $\mathcal{C}$ is (monoidally) equivalent to $\operatorname{Rep}(A)$, where $A$ is an odd-dimensional, semisimple quasi-Hopf algebra.

The following generalization of [13, Lemma 2.3] gives a linear improvement of the upper bounds in Algorithm 4.1 for a restricted class of integral modular categories and includes the MNSD setting as the special case $\ell=2$ :

Lemma 4.4. Suppose that $\mathcal{C}$ is an integral modular category of rank $n$ and there exist $k$ (weakly decreasing) integers $p_{1} \geq p_{2} \geq \cdots \geq p_{k}$ such that the non-trivial (isomorphisms classes of) simple objects can be partitioned into $k$ sets $P_{1}, \ldots, P_{k}$ such that $X \in P_{i}$ has $\operatorname{dim}(X)=p_{i}$ and $\left|P_{i}\right|=\ell$ for all $1 \leq i \leq k$. Then:

(i) the numbers $x_{i}:=\frac{\operatorname{dim} \mathrm{C}}{p_{i}^{2}}$ form a weakly increasing sequence of integers such that $\sum_{i=1}^{k} \frac{\ell}{x_{i}}+\frac{1}{x_{k+1}}=1$, and

(ii) $\ell i \leq x_{i} \leq(n+\ell-i \ell) \frac{A_{i}}{\ell}$ for $i \leq k$ and $(k+1) \leq x_{k+1} \leq \frac{A_{k+1}}{\ell}$, where $A_{1}=\ell$ and $A_{i}=A_{i-1}\left(A_{i-1}+1\right)$. 
Proof. The proof of (i) proceeds exactly as in [13, Lemma 2.3], so we focus on (ii). First define $x_{i}^{\prime}=x_{i}$ for $1 \leq i \leq k$ and $x_{k+1}^{\prime}=x_{k+1} / \ell$ so that

$$
\sum_{i=1}^{k+1} \frac{1}{x_{i}^{\prime}}=\frac{1}{\ell}
$$

and $x_{1}^{\prime} \leq \cdots \leq x_{k+1}^{\prime}=\ell x_{k+1}$. Observing that $n=k \ell+1$ we find that (ii) becomes $\ell i \leq x_{i}^{\prime} \leq(n+\ell-i \ell) \frac{A_{i}}{\ell}$. Since the $x_{i}^{\prime}$ are weakly increasing the lower bounds are clear: for if $\ell i>x_{i}^{\prime}$ for some $i$, then $\ell i>x_{i}^{\prime} \geq x_{i-1}^{\prime} \geq \cdots \geq x_{1}^{\prime}$, so $1 / \ell=i \frac{1}{\ell i}>\sum_{j=1}^{i} \frac{1}{x_{j}^{\prime}}$, a contradiction. For the upper bounds we follow the same strategy as in [13]: we use Takenouchi's [24] bound on the largest denominator $x_{k+1}^{\prime}$ in (4.2) and Landau's [14] estimate. In particular, if

$$
\sum_{i=1}^{k} \frac{1}{y_{i}}=r
$$

with $y_{1} \leq y_{2} \leq \cdots \leq y_{k}$, then Landau's result says that $y_{i} \leq(k-i+1) / r_{i-1}$, where $r_{0}=r$ and $r_{i}=r_{i-1}-1 / y_{i}$. Takenouchi's result says that the maximum denominator $y_{i}$ of a solution to $\frac{1}{\ell}=\sum_{i=1}^{k} \frac{1}{y_{i}}$ is $A_{k}$, where $A_{1}=\ell$ and $A_{i}:=$ $A_{i-1}\left(A_{i-1}+1\right)$. So in particular $1 / r_{i-1} \leq A_{i}$ for all $i$ and the result follows.

Lemma 4.4 provides a linear improvement on the bounds in [13, Lemma 2.3]: one has roughly $\operatorname{rank}(\mathcal{C}) / \ell$ variables as opposed to $\operatorname{rank}(\mathcal{C})$. Taking the case $\ell=2$ one can modify Algorithm 4.1 to search for possible MNSD modular categories in the obvious way. As the bounds are still doubly exponential the best result we can achieve with our (modified) algorithm is:

Theorem 4.5. All maximally non-self-dual modular categories of rank at most 11 are pointed.

Proof. The only non-trivial solution the modified algorithm produces is in rank 11, consisting of 9 simple objects of dimension 1 and two simple objects of dimension 3 . To eliminate this possibility we use a result in [11]: any integral modular category $\mathcal{C}$ is faithfully graded by its universal grading group $U(\mathcal{C})$, which is isomorphic to the group of invertible objects in $\mathcal{C}$. Since each component of a faithful grading must have the same FP-dimension, we see that this is impossible as $|U(\mathcal{C})|=9$ and any component $\mathcal{C}_{g}$ of the grading containing a simple object $X$ with $\operatorname{FPdim}(X)=3$ must have $\mathrm{FPdim}\left(\mathcal{C}_{g}\right) \geq 9$.

Remark 4.6. An obvious source of MNSD modular categories are those of the form $\operatorname{Rep}\left(D^{\omega} G\right)$, where $|G|$ is odd (that is, the representation category of the twisted double of a finite group of odd order). The smallest rank example of a non-pointed MNSD modular category we are aware of is the rank 25 category $\operatorname{Rep}\left(D\left(\mathbb{Z}_{3} \ltimes \mathbb{Z}_{7}\right)\right)$ of dimension 441 , where $\mathbb{Z}_{3} \ltimes \mathbb{Z}_{7}$ is a non-abelian semidirect product. We remark that it is possible that for $G$ abelian, $\operatorname{Rep}\left(D^{\omega} G\right)$ can be non-pointed; see [16]. However, for $|G|$ odd, such examples do not have rank less than 25. One might speculate that any MNSD modular category of rank at most 23 is pointed. 
We close with the following related question that we find interesting:

Question 4.7. Is there an odd-dimensional modular category that is non-grouptheoretic?

\section{Conclusions And Future Directions}

We have made significant progress towards classifiying modular categories with twist matrices of order 2,3,4 or 6 . It is not clear how to extend these techniques to other orders. One might hope that one could generalize [17, Theorem 8.4] to non-integral categories to get some statement about the possible prime divisors of $\mathrm{FPdim}(\mathcal{C})$ in terms of those of the order of the twist matrix. However, the relevant number field would no longer be $\mathbb{Z}$ so that other complications would arise, particularly concerning units.

For rank greater than 11 even the improved bounds for MNSD modular categories become too large for our computational techniques. However, if one considers MNSD modular categories with:

(1) rank $r$,

(2) $\operatorname{FPdim}\left(X_{i}\right) \leq M$, for each simple $X_{i}$, and

(3) $\left|\left\{\operatorname{FPdim}\left(X_{i}\right): 0 \leq i \leq r-1\right\}\right|=t\left(X_{i}\right.$ simple),

further headway can be made. For example, taking $r \leq 23, M=45$ and $t \leq 6$ we set $k_{i}=\left|\left\{j: \operatorname{FPdim}\left(X_{j}\right)=d_{i}\right\}\right|$ with $d_{0}=1$ and we obtain (using [6. Lemma 1.2] and [7, Prop. 8.15]):

$$
\begin{aligned}
& k_{0} \equiv 1 \quad \bmod 2, \\
& k_{i} \equiv 0 \quad \bmod 2(i \neq 0), \\
& \sum_{i=0}^{t-1} k_{i}=r, \\
& \sum_{j \neq i} k_{j}\left(d_{j}\right)^{2} \equiv 0 \quad \bmod \left(d_{i}\right)^{2}, \\
& \sum_{j \neq 0} k_{j}\left(d_{j}\right)^{2} \equiv 0 \quad \bmod k_{0} .
\end{aligned}
$$

For any fixed $r \leq 23$ and subsets of $\{d: 3 \leq d \leq 45, d \in 2 \mathbb{Z}+1\}$ of size at most 5 we convert these congruences to a system of linear Diophantine equations and then use the Smith normal form of the corresponding matrix to solve for the $k_{i}$. Applying further classification theorems and ad hoc techniques produces the following partial result:

Theorem 5.1. If $\mathcal{C}$ is a non-pointed maximally non-self-dual modular category with $13 \leq \operatorname{rank}(\mathcal{C}) \leq 23$, then either $\mathcal{C}$ has:

(a) $\operatorname{FPdim}\left(X_{i}\right) \geq 47$ for some simple $X_{i}$ or

(b) $\left|\left\{\operatorname{FPdim}\left(X_{i}\right): 0 \leq i \leq m-1\right\}\right| \geq 7$.

This is further evidence that perhaps a non-pointed MNSD modular category of smallest rank is indeed the category of rank 25 and dimension 441 described above.

More generally, one can also obtain partial classification results for integral modular categories of small rank by bounding the FP-dimension further (below the 
doubly-exponential bound of $u_{n}$ in Algorithm 4.1). For example, using a streamlined version of Algorithm 4.1 provided by K. Rusek and some classification theorems we can prove:

Theorem 5.2. If a non-pointed modular category $\mathcal{C}$ has rank 7 , then $\operatorname{FPdim}(\mathcal{C})>$ $10^{5}$.

\section{ACKNOWLEDGEMENTS}

The authors benefitted from discussions with K. Rusek, M. Papanikolas, Y. Sommerhäuser and D. Naidu. The second author was partially supported by NSA grant H98230-10-1-0215.

\section{REFERENCES}

[1] B. Bakalov and A. Kirillov, Jr., Lectures on tensor categories and modular functors, University Lecture Series, vol. 21, Amer. Math. Soc., Providence, RI, 2001. MR.1797619 (2002d:18003)

[2] M. Cuntz, Integral modular data and congruences, J. Algebraic Combin. 29 (2009), 357-387. MR2496312 (2010b:20024)

[3] D. R. Curtiss, On Kellogg's diophantine problem, Amer. Math. Monthly 29 (1922), 380-387.

[4] V. Drinfeld, S. Gelaki, D. Nikshych, and V. Ostrik, Group-theoretical properties of nilpotent modular categories (2010). arXiv:0704.0195

[5] _ On braided fusion categories I, Selecta Math. 16 (2010), no. 1, 1-119. MR2609644 (2011e:18015)

[6] P. Etingof and S. Gelaki, Some properties of finite-dimensional semisimple Hopf algebras, Math. Res. Lett. 5 (1998), no. 1-2, 191-197. MR1617921 (99e:16050)

[7] P. Etingof, D. Nikshych, and V. Ostrik, On fusion categories, Ann. of Math. (2) 162 (2005), no. 2, 581-642. MR2183279 (2006m:16051)

[8] - Weakly group-theoretical and solvable fusion categories, Adv. Math. 226 (2011), no. 1, 176-205. MR2735754

[9] T. Gannon, Modular data: The algebraic combinatorics of conformal field theory, J. Algebraic Combin. 22 (2005), 211-250. MR2164398(2006h:81254)

[10] S. Gelaki, D. Naidu, and D. Nikshych, Centers of graded fusion categories, Algebra Number Theory 3 (2009), no. 8, 959-990. MR2587410 (2011b:18011)

[11] S. Gelaki and D. Nikshych, Nilpotent fusion categories, Adv. Math 217 (2008), no. 3, 10531071. MR2383894 (2009b:18015)

[12] R. Guy, Unsolved problems in number theory, 3rd ed., Problem Books in Mathematics, Springer, 2004. MR2076335 (2005h:11003)

[13] S.-M. Hong and E. Rowell, On the classification of the Grothendieck rings of non-self-dual modular categories, J. Algebra 324 (2010), no. 5, 1000-1015. MR2659210 (2011k:18018)

[14] E. Landau, Über die Klassenzahl der binären quadratischen Formen von negativer Discriminante, Math. Ann. 50 (1903), 671-676.

[15] D. Naidu and E. C. Rowell, A finiteness property for braided fusion categories, Algebr. Represent. Theory 14 (2011), no. 5, 837-855.

[16] S.-H. Ng, Non-commutative, non-cocommutative semisimple Hopf algebras arise from finite abelian groups, Groups, rings, Lie and Hopf algebras (St. John's, NF, 2001), Math. Appl. 555, Kluwer Acad. Publ., Dordrecht, 2003, pp. 167-177. MR.1995058 (2004h:16037)

[17] S.-H. Ng and P. Schauenburg, Frobenius-Schur indicators and exponents of spherical categories, Adv. Math. 211 (2007), no. 1, 34-71. MR2313527 (2008b:16067)

[18] _ Congruence subgroups and generalized Frobenius-Schur indicators, Comm. Math. Phys. 300 (2010), 1-46. MR2725181

[19] Online encyclopedia of integer sequences, 2010. http://www.research.att.com/njas/ sequences/.

[20] V. Ostrik, Fusion categories of rank 2, Math. Res. Lett. 10 (2003), no. 2-3, 177-183. MR.1981895 (2004c:18015)

[21] Pre-modular categories of rank 3, Mosc. Math. J. 8 (2003), no. 1, 111-118. MR2422269 (2010f:18006) 
[22] E. C. Rowell, From quantum groups to unitary modular tensor categories, Contemp. Math. 413, Amer. Math. Soc., Providence, RI, 2006, 215-230. MR2263097 (2007i:18007)

[23] E. Rowell, R. Stong, and Z. Wang, On classification of modular tensor categories, Comm. Math. Phys. 292 (2009), no. 2, 343-389. MR2544735 (2011b:18013)

[24] T. Takenouchi, On an indeterminate equation, Proceedings of the Physico-Mathematical Society of Japan 3 (1921), 78-92.

[25] V. Turaev, Modular categories and 3-manifold invariants, Internat. J. Modern Phys. B 6 (1992), no. 11-12, 1807-1824. MR1186845(93k:57040)

Department of Mathematics, Texas A\&M University, College Station, Texas 77843

E-mail address: paul.bruillard@math.tamu.edu

Department of Mathematics, Texas A\&M University, College Station, Texas 77843

E-mail address: rowell@math.tamu.edu 